\title{
High expression levels of erythropoietin and its receptor are not correlated with shorter survival in human glioblastoma
}

\author{
J. BRUNOTTE ${ }^{1}$, H.C. BOCK ${ }^{2}$, W. BRÜCK ${ }^{3}$, B. HEMMERLEIN ${ }^{4 *}$ and H. STRIK ${ }^{1,5^{*}}$ \\ Departments of ${ }^{1}$ Neurology, ${ }^{2}$ Neurosurgery, ${ }^{3}$ Neuropathology, and ${ }^{4}$ Pathology, University Medicine Göttingen, \\ Göttingen; ${ }^{5}$ Department of Neurology, University of Marburg, Marburg, Germany
}

Received September 14, 2010; Accepted December 29, 2010

DOI: $10.3892 /$ etm.2011.198

\begin{abstract}
Erythropoietin (EPO) is used to treat anemia in neoplastic disease. EPO also exerts neuroprotective effects on neuronal cells, making a prophylactic use against the neurocognitive effects of radiochemotherapy probable. However, EPO/ EPO-receptor (EPOR) signalling has been also detected in glioblastoma cells. Data collected in vitro and in vivo show conflicting results on the effect of EPO on malignant gliomas. The association between EPO and EPOR expression and the prognosis of human glioblastomas was analyzed. Probes of human glioblastomas with complete documentation of clinical course and treatment were assessed by immunohistochemistry for the expression of EPO and EPOR $(n=80)$. Using univariate and multivariate survival analysis, the association with age, gender, radiation, chemotherapy and extent of resection was determined. High levels of EPOR were correlated with a median survival advantage of 7 months $(\mathrm{p}<0.01)$. By univariate, but not multivariate, analysis, high levels of EPO and EPOR were associated with a significant prolongation of 7 months median survival when compared to low levels of both molecules. In patients treated with radiochemotherapy adjuvant to surgery, the median survival was 6.5 months longer in patients with high levels of EPOR $(\mathrm{p}<0.04)$. According to previous studies, longer patient survival is associated with EPOR expression. Therefore, EPO appears to be safe for the treatment of anemia in glioblastoma patients. However, a prophylactic use, i.e., for neuroprotection, is not recommended in light of the functional studies described in the literature.
\end{abstract}

\section{Introduction}

Glioblastoma is one of the most malignant forms of brain tumors and is associated with a poor median patient survival of

Correspondence to: Dr Herwig Strik, Department of Neurology, University of Marburg, Rudolf Bultmann Str. 8, D-35039 Marburg, Germany

E-mail: strik@med.uni-marburg.de

*Contributed equally

Key words: erythropoietin, erythropoietin receptor, glioblastoma, prognosis, survival approximately 12 months in unselected series (1). In selected patients, multimodal treatment with radiochemotherapy achieves prolonged survival times of more than 2 years in $25 \%$ of the patients (2). However, extended use of chemotherapy may lead to myelosuppression, including anemia, which may require the application of growth factors. Moreover, cognitive impairment may result from glioma treatment, raising the question whether neuroprotective agents are useful during combined radiochemotherapy (3).

Erythropoietin (EPO) is a glycoprotein hormone primarily known as a regulator of erythropoiesis. EPO-mRNA codes for a pre-pro-protein with 193 amino acids. During posttranslational modification, 27 amino acids are cut at the $\mathrm{N}$-terminal and arginin at the $\mathrm{C}$-terminal residue through an intracellular carboxypeptidase, which results in the 165-amino acid EPO protein $(4,5)$.

EPO-receptor (EPOR) is a cytokine receptor. Its extracellular domain is similar to the receptors for other cytokines, such as G-CSF or GM-CSF (6). In erythropoietic precursor cells, three different splice variants have been identified: fulllength EPOR (f-EPOR), truncated EPOR (t-EPOR) and soluble EPOR (s-EPOR) (7). All forms have the same extracellular binding domain, but f-EPOR and s-EPOR have shortened cytoplasmic and transmembranous components, respectively. The distinct role of these various forms has yet to be elucidated.

The role of EPO in cancer and its possible therapeutic use in the management of side effects of neoplastic disease itself and the effects of anticancer treatment is being debated. EPO is used to treat anemia associated with neoplastic disease itself or with antineoplastic chemotherapy (8). Moreover, EPO has been shown to exert neuroprotective potential during radiotherapy through EPOR expression on cerebral neurons $(3,9)$. On the other hand, EPOR has also been detected on glioma cells (10), raising concern about the safety of EPO treatment in glioma patients (11).

In the present study, we assessed the expression of EPO and EPOR in human glioblastomas. The results were correlated with overall patient survival with respect to age, extent of resection and antineoplastic treatment. In selected cases, the expression of both proteins in primary and recurrent tumors was compared.

\section{Patients and methods}

Tumor bank. Cases of glioblastoma, for which the complete clinical course was documented and enough paraffin-embedded 
tissue was available for immunohistochemistry were collected. Material was available in the archives of the Department of Neuropathology from patients that underwent surgery between 1998 and 2005 at the Department of Neurosurgery of the University of Göttingen.

The patients were assigned to 5 treatment groups. The first group of 36 patients underwent tumor resection only; 10 patients received radiotherapy and adjuvant sequential chemotherapy with ACNU and temozolomide $\left(150-200 \mathrm{mg} / \mathrm{m}^{2}\right.$, days $1-5 / 28) ; 8$ patients received temozolomide concomitantly to radiotherapy $\left(75 \mathrm{mg} / \mathrm{m}^{2}\right)$ followed by six cycles of adjuvant temozolomide (150-200 mg/m², days 1-5/28); 14 patients received adjuvant ACNU after radiotherapy; and 22 patients were treated with adjuvant temozolomide only $\left(150-200 \mathrm{mg} / \mathrm{m}^{2}\right.$, days 1-5/28) after radiotherapy.

Immunohistochemistry. For immunohistochemical staining, the following antibodies were used: rabbit anti-ErythropoetinR (C-20) sc-695 and goat anti-Erythropoetin (N-19) sc-1310, (both from Santa Cruz Biotechnology, Inc., USA). The bridge antibody was AffiniPure rabbit anti-goat, lot 77186 (Dianova). EnVision-reagent included in the Dako Real ${ }^{\mathrm{TM}}$ EnVision $^{\mathrm{TM}}$ Detection system, Peroxidase/DAB ${ }^{+}$, rabbit/mouse, code K5007 (Dako, Denmark).

Paraffin-embedded tissue blocks were cut into 2- to $4-\mu \mathrm{m}$ slices and dried overnight at $37^{\circ} \mathrm{C}$. Sections were deparaffinized in xylol and ethanol and washed in demineralized water. Subsequently, endogenous peroxidase was blocked with $\mathrm{H}_{2} \mathrm{O}_{2}$ for $20 \mathrm{~min}$. Following application of Tris to buffer $\mathrm{pH}$ unspecific binding was blocked with BSA for EPOR only, since cross-reaction with the bridge antibody was observed with the EPO protein.

The primary antibody was applied at a concentration of $2 \mu \mathrm{g} / \mathrm{ml}$ for $60 \mathrm{~min}$. The EPOR antibody was diluted with Tris-buffer and EPO protein antibody with Tris-buffer plus Tween reagent 1:200 in order to reduce surface tension and improve distribution of the antibody. Following $60 \mathrm{~min}$ of incubation, the sections were washed twice with Tris buffer.

Since the Dako Real EnVision ${ }^{\mathrm{TM}}$ detection system only reacts with mouse or rabbit antibodies, an additional rabbitgoat bridging antibody was applied at $4.5 \mu \mathrm{l} / \mathrm{ml}$ to enable binding of the goat anti-EPO antibody and incubated for $30 \mathrm{~min}$.

EnVision reagent from the Dako Real EnVision detection system was used at $100 \mu \mathrm{l}$ for $30 \mathrm{~min}$ as a secondary antibody to specifically bind to the Fc-fragments of the EPOR antibody or the bridging antibody for the EPO protein.

To visualise the marked antigen, the color reagent was synthesised with a mixture of Dako Real ${ }^{\mathrm{TM}}$ Substrate Buffer and Dako Real ${ }^{\mathrm{TM}} \mathrm{DAB}^{+}$Chromogen (concentrated x50) at 50:1. The solution was applied at $100 \mu \mathrm{l}$ per slice in a dark wet chamber for $20 \mathrm{~min}$ and then washed four times with demineralized water. The slices were then stained with hematoxylin for $2 \mathrm{~min}$ and running water for $1 \mathrm{~min}$ and then fixed with Cytoseal 60 (Richard Allen Scientific).

For the positive controls, sections of normal human kidney tissue were stained using the protocol described. Sections of glioblastomas and kidney were treated as described, without primary antibody in the Tris-buffer, to serve as negative controls using the same staining procedure.
Morphometry. The stained sections were assessed with a regular light microscope at x100 and x400 magnification, with the help of a counting net. For each section, the frequency of stained cells was assessed in 6-8 high-power fields depending on the size of the probe, and a semiquantitative score was determined: score $1,<5 \%$ positive cells; score $2,5-20 \%$ positivity; score $3,21-50 \%$ positivity; score $4,>50 \%$ positivity. For statistical analysis, the mean value of all single scores multiplied by 10 was calculated as the cumulative score, resulting in values from 0 to 40 .

Statistical analysis. The data were analyzed with the statistic computer program Statistica v8.1 based on a significance of $\mathrm{p} \leq 0.05$. The Gehan's Wilcoxon test was used to analyze overall survival in groups divided by age, gender and expression scores. The Chi-square test was used to assess the survival in therapy groups and groups of high or low protein and receptor expression. A probable correlation between gender and expression scores was assessed using the MannWhitney U test. A probable correlation between age and expression scores and between the expression of EPO and EPOR was analyzed with the Spearman rank correlation test. Multivariate analysis, stratified analysis and comparison of sum scores EPO + EPOR were carried out with the Cox regression model. The comparison between expression scores of initial and recurrent tumors was performed with the Wilcoxon signed-rank test.

\section{Results}

For immunohistochemical staining, 107 paraffin-embedded probes were collected: 89 primary tumors from 52 male and 37 female patients, of which 13 first and 5 secondary relapses were available. The cases were diagnosed neuropathologically as glioblastomas WHO-grade IV, except 2 cases of gliosarcoma. The median age of patients at diagnosis was 63 years (range 30-81). Only 1 patient was alive at 115 months from initial diagnosis at the time of analysis. The median survival of the unselected patients was 9 months ( \pm SD 18.2). Patients $\leq 63$ years old had a mean survival of 13 months, while patients at an older age were associated with a shorter survival of only 5 months $(\mathrm{p}<0.0001)$ (Table I). Patients in the surgery only group $(n=36)$ had an average survival of 3.6 months. Patients receiving adjuvant temozolomide after radiotherapy $(n=21)$ had a mean survival of 16.1 months, while patients receiving concomitant and adjuvant temozolomide $(n=8)$ had a mean survival of 22.1 months. Patients receiving adjuvant ACNU $(n=14)$ were associated with an average patient survival of 23.1 months, and patients receiving concomitant plus adjuvant temozolomide followed by adjuvant ACNU in second line therapy $(n=10)$ achieved a mean survival of 25.9 months. The difference in survival times between the surgery only group and the chemotherapy groups in this retrospective, not randomized series was statistically different $(\mathrm{p}<0.0001)$. The analysis of the extent of resection showed a clear benefit for patients with gross total resection $(n=45)$ who achieved a median survival of 13 months ( \pm SD 23.2), while partial resection $(n=33)$ was only associated with a survival of 6 months ( \pm SD 8.6) and biopsy only with 3 months ( \pm SD 6.2). 
Table I. Median survival in patients with high or low expression levels of EPO or EPOR.

\begin{tabular}{lcc}
\hline & p-value & Median survival advantage with high EPO or EPOR \\
\hline EPO high vs. low & 0.08 & 6 months, not significant \\
EPOR high vs. low & $<0.01$ & 7 months \\
EPO (radiochemotherapy) & Not significant & No advantage \\
EPOR (radiochemotherapy) & 0.04 & 6.5 months \\
EPO (younger age) & Not significant & No advantage \\
EPOR (younger age) & 0.02 & 9 months \\
Epo multivariate analysis & Not significant & No advantage \\
EpoR multivariate analysis & Not significant & No advantage \\
Cumulative EPO + EpoR multivariate analysis & 0.07 & No advantage \\
Cumulative EPO + EpoR & 0.06 & 6.5 months, not significant \\
\hline
\end{tabular}

EPO and EPOR. In some of the probes, there was insufficient tumor tissue for a statistical analysis of EPO and EPOR. Therefore, 9 probes had to be excluded. For the analysis presented here, 80 probes of first and 13 probes of secondary resections were included.

Staining with both EPO and EPOR exhibited an inhomogeneous pattern with preponderance to perinecrotic areas, mitotic or multinucleated giant cells and microvascular endothelial proliferates (data not shown).

The median overall survival of the patients in the EPO and EPOR group was 9 months, the percentage of long-term survivors over 2 years was $14 \%$. A total of 11 patients (14\%) survived less than 4 weeks (ultrashort-term survivors).

Of the 80 patients in this group, $51 \%$ underwent macroscopically total resection, $38 \%$ partial resection and $11 \%$ biopsy only.

EPOR score. The median score for EPOR in 80 probes was 31 (19-40). The patients were divided into groups based on scores lower $(n=30)$ or higher and equal to $(n=50)$ the median score.

Low EPOR scores were observed in 36\% of the female and in $38 \%$ of the male patients, and high scores in $64 \%$ of the females and in $62 \%$ of the males.

The median age in the low-EPOR group was 67 years and in the high-EPOR group 62.5 years.

A total of $47 \%$ of patients in the low-EPOR and $54 \%$ in the high-EPOR group underwent gross total resection, while 53\% in the low-score and $46 \%$ in the high-score group received partial resection or biopsy only. A total of $53 \%$ of the patients in the low-score and $32 \%$ in the high-score EPOR group underwent surgery only, while $47 \%$ patients in the low-score and $68 \%$ in the high-score group received chemotherapy in addition to radiotherapy.

EPO score. The overall positivity of EPO protein was higher than its receptor.

Patients were divided according to scores lower $(n=40)$ or higher and equal $(n=40)$ to the median score of 35 .

A total of $52 \%$ of the female and $49 \%$ of the male patients were assigned to the low-score group. The median age in the low-score group was 65 years, and the median age in the highscore group was 60.5 years.

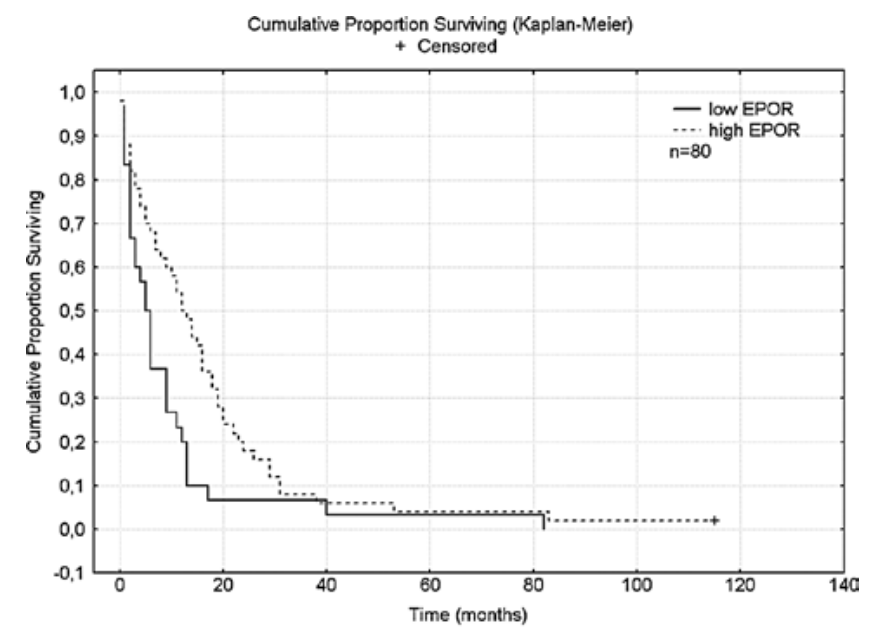

Figure 1. Overall survival and expression levels of EPOR. Patients with high levels had a significantly longer survival as assessed by univariate analysis $(\mathrm{p}<0.01$, Kaplan-Meier).

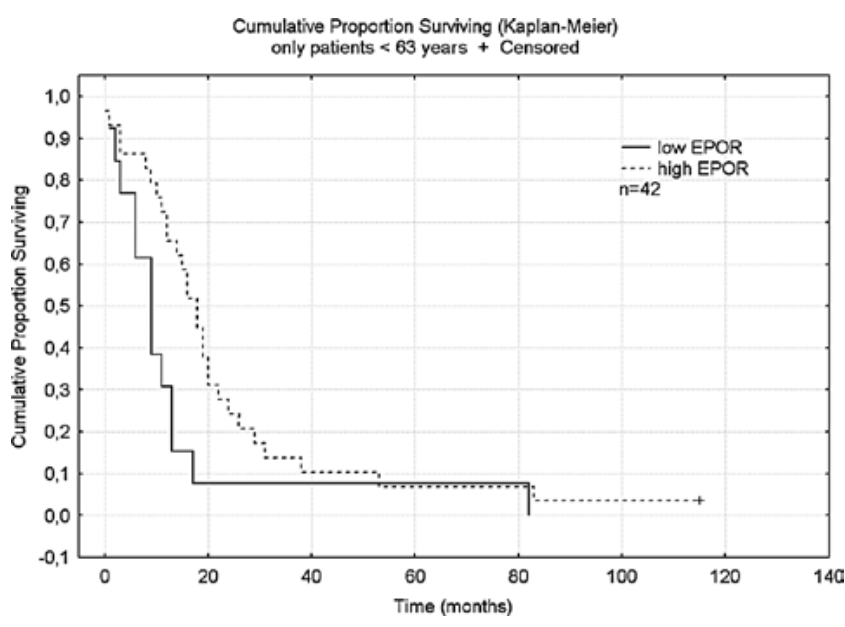

Figure 2. Overall survival of patients younger than 63 years of age. Patients with high levels of EPOR had a significant survival benefit of 9 months $(\mathrm{p}<0.02$, Kaplan-Meier).

Gross total resection was possible in $45 \%$ of the patients in the low-score group and in $57.5 \%$ of patients in the high-score group. Surgery only was performed in $47.5 \%$ of the patients in 


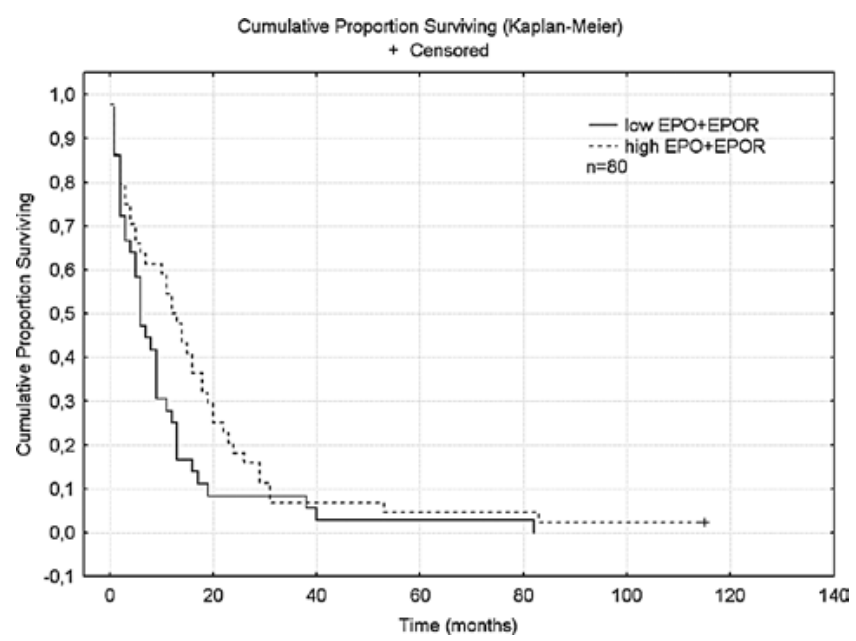

Figure 3. Overall survival and cumulative EPO + EPOR. Patients with high expression levels of EPO + EPOR had a survival benefit of 6.5 months; this just failed to reach significance ( $\mathrm{p}=0.058$; Kaplan-Meier plot).

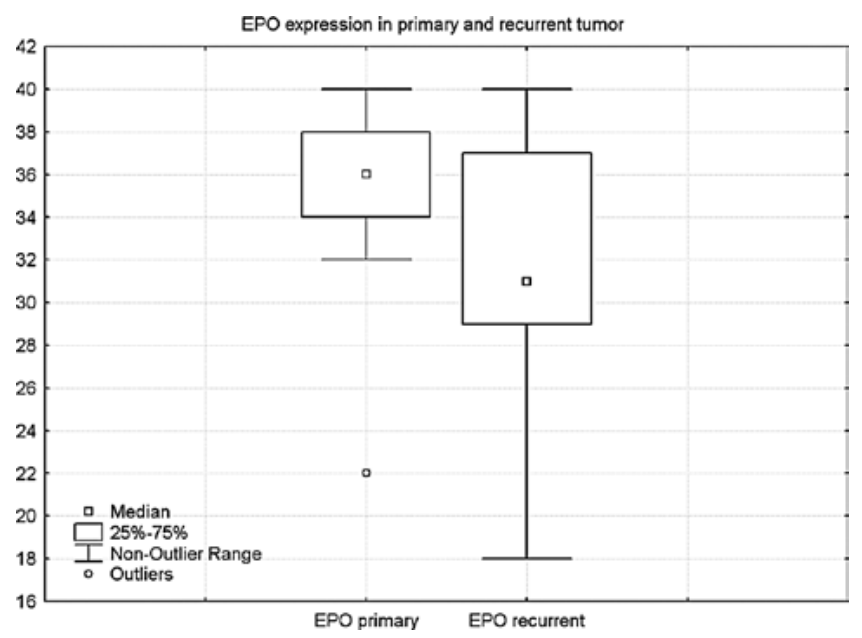

Figure 4. EPO levels were significantly lower in recurrent as compared to primary tumors $(\mathrm{p}=0.05, \mathrm{n}=13)$.

the low- and 32.5\% in the high-score EPO group, while adjuvant chemotherapy was administered to $52.5 \%$ in the low- and to $67.5 \%$ in the high-score group.

Correlation analyses. No correlation was found between EPO or EPOR and gender or age. A highly significant correlation, however, was found between EPO protein and its receptor EPOR $(\mathrm{p}<0.000001)$.

EPO, EPOR and overall survival. The median survival for patients with high expression of EPOR was 12.5 months; significantly longer than that for patients with low expression (5.5 months, p<0.01) (Fig. 1). The median survival for patients with high expression of EPO protein was 12 months and that for patients with low expression of EPO protein was 6 months. The advantage of overall survival, however, was not significant $(\mathrm{p}=0.08)$.

The median survival of patients treated with radiochemotherapy $(n=48)$ with high and low expression of EPOR was 18 and 11.5 months, respectively $(\mathrm{p}<0.04)$.
Patients younger than the median age of 63 years $(n=42)$ with high expression had a mean survival of 18 months, which was significantly longer than patients in the same age group with lower expression of EPOR who had a median survival of 9 months $(\mathrm{p}<0.02)$ (Fig. 2). No difference in survival was noted in patients with different EPO expression $(p=0.10)$. In separately assessed older patients, EPO or EPOR had no influence on survival.

Multivariate regression analysis showed the significant influence of extent of resection $(\mathrm{p}=0.005)$, age $(\mathrm{p}=0.03)$ and type of chemotherapy $(\mathrm{p}=0.0002)$. The influence of EPOR on survival failed to remain significant by multivariate analysis $(\mathrm{p}=0.096)$. Likewise, in the EPO group, the extent of resection $(p=0.02)$, age $(p=0.04)$ and type of chemotherapy $(p=0.00005)$ had a significant impact on the overall survival, while the expression of EPO protein was not significant ( $\mathrm{p}=0.133$ ).

None of the analyses using the Cox proportional hazard model and stratifying by age, extent of resection or adjuvant chemotherapy revealed a significant influence of EPO or EPOR expression on survival. This may indicate an intercorrelation effect between the scores of expression and the extent of resection, age or type of chemotherapy, probably due to an imbalanced distribution of high and low expression of EPO and EPOR in the subgroups of resection, age and chemotherapy.

When EPO and EPOR were added for a cumulative score, a longer survival of 12.5 months was found for patients with high cumulative scores as compared to 6 months for patients with low scores; this result just failed to reach significance ( $p=0.058)$ (Fig. 3). This result also failed to be significant in the multivariate analysis, including age, extent of resection and adjuvant therapy $(\mathrm{p}=0.07)$.

The analysis of EPOR in 13 paired tumors showed a mean score of 32.7 in primary and 29.6 in recurrent tumors, a difference which was not significant.

In contrast, in 13 pairs tested for EPO protein, a significant difference was found between the mean score of 35.1 in primary and 31.5 in recurrent tumors ( $\mathrm{p}=0.05)$ (Fig. 4).

\section{Discussion}

The present study assessed the impact of EPO and EPOR expression on the prognosis of patients with glioblastoma. No negative association between EPO or EPOR and survival was found, but rather a trend towards prolonged survival in certain subgroups. These findings indicate that the therapeutic application of EPO may not have a negative effect on glioma growth in vivo.

EPO is used for various indications in neoplastic disease. Anemia, caused by systemic cancer or chemotherapy, may require the use of hematopoietic growth factors as a symptomatic treatment (8). The tumor itself may be resistant to apoptosis-inducing stimuli such as ionizing irradiation or chemotherapy through intratumoral hypoxia, which is a frequent condition of solid tumors (12). An increased hemoglobin level increases intratumoral oxygenation (13), which may lead to improved response to radiation and chemotherapy $(14,15)$. Therefore, EPO has been used in various studies to increase tumor oxygenation in order to augment the effect of antineoplastic therapy (16-18). A specific indication during 
brain tumor treatment may protect against the cognitive impairment caused by radiochemotherapy. Neuroprotective effects of EPO have been shown to be exerted via EPOR expressed in cerebral neurons during radiotherapy $(3,9)$.

Initial concerns concerning the use of EPO have been raised by the detection of EPOR in glioma cells $(10,19)$. In functional studies, EPO protected cultured glioblastoma cells from cisplatin cytotoxicity and promoted their invasiveness (11). Hassouna et al observed no growth-stimulating effect of EPO in cell culture and no growth promotion in experimental mouse glioma (20). Resistance to induction of apoptosis by ionizing irradiation and temozolomide, however, was found to increase in 3 of 4 cell lines when EPO was applied concomitantly. An initial immunohistochemical study revealed no negative impact of EPOR expression on glioma cells. In the subgroup of younger patients, a more favorable survival was found to be associated with higher levels of EPOR (21). In the present study, EPO protein was analyzed in addition to its receptor. Moreover, we assessed the postoperative treatment and thus were able to include relevant clinical prognostic factors into our analysis. In line with the results of Mittelbronn et al, a positive correlation was found between EPOR and survival particularly in younger patients. Moreover, higher levels of EPO were also associated with better survival. In light of the in vitro results of Hassouna et al who found an increased resistance to radiochemotherapy, we assessed the impact of EPO and EPOR in patients receiving radiochemotherapy and found that higher levels of EPOR tended to be associated with longer survival $(\mathrm{p}<0.04)$. Notably, we observed a significant trend towards reduced levels of EPO in recurrent as opposed to primary tumors. Although this trend was not significant for EPOR in this small group, the progression to more resistant recurrent tumors was rather associated with a reduction than with increased activity of the EPO-EPOR system.

These immunohistochemical results do not indicate a negative effect of EPO on glioma growth in vivo. This finding, however, is not sufficient to propagate the broad prophylactic use of EPO, i.e., for neuroprotection during glioma treatment. In various systemic tumors, EPO was applied to sensitize tumors for radiation or chemotherapy. While certain studies observed beneficial effects (16-18), a lower survival rate was found in head-and-neck and breast cancer $(22,23)$. Apart from unclear effects on the tumor growth itself, systemic side effects including deep venous thrombosis or arterial hypertension may have worsened the patient outcome (24).

Based on the data presented here, symptomatic treatment of anemia in glioma patients with EPO appears to be safe and without the risk of accelerated tumor growth. A prophylactic use of EPO aimed at neuroprotection or at enhancing the effect of adjuvant therapy is not recommended with respect to the results of functional studies. Further experiments are required to address this issue.

\section{References}

1. Davis FG, McCarthy BJ, Freels S, Kupelian V and Bondy ML: The conditional probability of survival of patients with primary malignant brain tumors: surveillance, epidemiology, and end results (SEER) data. Cancer 85: 485-491, 1999.
2. Stupp R, Mason WP, van den Bent MJ, et al: Radiotherapy plus concomitant and adjuvant temozolomide for glioblastoma. $\mathrm{N}$ Engl J Med 352: 987-996, 2005.

3. Erbayraktar S, de Lanerolle $\mathrm{N}$, de Lotbiniere A, et al: Carbamylated erythropoietin reduces radiosurgically-induced brain injury. Mol Med 12: 74-80, 2006.

4. Mulcahy L: The erythropoietin receptor. Semin Oncol 28: 19-23, 2001.

5. Sasaki R, Masuda S and Nagao M: Erythropoietin: multiple physiological functions and regulation of biosynthesis. Biosci Biotechnol Biochem 64: 1775-1793, 2000.

6. Leyland-Jones B: Evidence for erythropoietin as a molecular targeting agent. Semin Oncol 29: 145-154, 2002.

7. Nakamura Y, Komatsu $\mathrm{N}$ and Nakauchi H: A truncated erythropoietin receptor that fails to prevent programmed cell death of erythroid cells. Science 257: 1138-1141, 1992.

8. Khan FA, Shukla AN and Joshi SC: Anaemia and cancer treatment: a conceptual change. Singapore Med J 49: 759-764, 2008.

9. Smith RE Jr: Erythropoietic agents in the management of cancer patients. Part 2: studies on their role in neuroprotection and neurotherapy. J Support Oncol 2: 39-49, 2004.

10. Berdel WE, Oberberg D, Reufi B and Thiel E: Studies on the role of recombinant human erythropoietin in the growth regulation of human nonhematopoietic tumor cells in vitro. Ann Hematol 63: 5-8, 1991

11. Mohyeldin A, Dalgard CL, Lu H, et al: Survival and invasiveness of astrocytomas promoted by erythropoietin. J Neurosurg 106: 338-350, 2007.

12. Molls M, Stadler P, Becker A, Feldmann HJ and Dunst J: Relevance of oxygen in radiation oncology. Mechanisms of action, correlation to low hemoglobin levels. Strahlenther Onkol 174: 13-16, 1998.

13. Kelleher DK, Mattheinsen U, Thews O and Vaupel P: Blood flow, oxygenation, and bioenergetic status of tumors after erythropoietin treatment in normal and anemic rats. Cancer Res 56: 4728-4734, 1996.

14. Brizel DM, Sibley GS, Prosnitz LR, Scher RL and Dewhirst MW: Tumor hypoxia adversely affects the prognosis of carcinoma of the head and neck. Int J Radiat Oncol Biol Phys 38: 285-289, 1997.

15. Höckel M, Schlenger K, Aral B, Mitze M, Schaffer U and Vaupel P: Association between tumor hypoxia and malignant progression in advanced cancer of the uterine cervix. Cancer Res 56: 4509-4515, 1996.

16. Ning S, Hartley C, Molineux G and Knox SJ: Darbepoietin alpha potentiates the efficacy of radiation therapy in mice with corrected or uncorrected anemia. Cancer Res 65: 284-290, 2005.

17. Pinel S, Barberi-Heyob M, Cohen-Jonathan E, Merlin JL, Delmas C, Plenat F and Chastagner P: Erythropoietin-induced reduction of hypoxia before and during fractionated irradiation contributes to improvement of radioresponse in human glioma xenografts. Int J Radiat Oncol Biol Phys 59: 250-259, 2004.

18. Thews O, Koenig R, Kelleher DK, Kutzner J and Vaupel P: Enhanced radiosensitivity in experimental tumours following erythropoietin treatment of chemotherapy-induced anaemia. $\mathrm{Br}$ J Cancer 78: 752-756, 1998

19. Westphal G, Niederberger E, Blum C, et al: Erythropoietin and G-CSF receptors in human tumor cells: expression and aspects regarding functionality. Tumori 88: 150-159, 2002.

20. Hassouna I, Sperling S, Kim E, et al: Erythropoietin augments survival of glioma cells after radiation and temozolomide. Int J Radiat Oncol Biol Phys 72: 927-934, 2008.

21. Mittelbronn M, Capper D, Bunz B, et al: De novo erythropoietin receptor (EPO-R) expression in human neoplastic glial cells decreases with grade of malignancy but is favourably associated with patient survival. Neuropathol Appl Neurobiol 33: 299-307, 2007.

22. Henke M, Laszig R, Rube C, et al: Erythropoietin to treat head and neck cancer patients with anaemia undergoing radiotherapy: randomised, double-blind, placebo-controlled trial. Lancet 362: 1255-1260, 2003

23. Leyland-Jones B, Semiglazov V, Pawlicki M, et al: Maintaining normal hemoglobin levels with epoetin alfa in mainly nonanemic patients with metastatic breast cancer receiving first-line chemotherapy: a survival study. J Clin Oncol 23: 5960-5972, 2005.

24. Bohlius J, Wilson J, Seidenfeld J, et al: Recombinant human erythropoietins and cancer patients: updated meta-analysis of 57 studies including 9353 patients. J Natl Cancer Inst 98: 708-714, 2006. 